\title{
Analysis of K-Connected MANETs for QoS Multicasting using EDMSTs Based on Connectivity Index
}

\author{
Arun Kumar B. R \\ MCA Dept., Sir MVIT, \\ Bangalore, Karnataka \& \\ Dept. of CS, School of Science \\ \& Technology, Dravidian \\ University, Kuppam - A.P, India.
}

\author{
Lokanatha C. Reddy \\ Dept. of CS, School of Science \\ \& Technology, Dravidian \\ University, Kuppam- A.P, India.
}

\author{
Prakash S. Hiremath \\ Dept. of P.G Studies \& \\ Research Group, Gulbarga \\ University, Gulbarga, \\ Karnataka, India
}

\begin{abstract}
The survivability of the network is closely related to the connectivity of the network. For many network applications such as a sensor network/ad hoc network for battlefield monitoring or wild fire tracking etc., survivability is one of most important requirements, and therefore a number of related problems are proposed. In this paper we study the survivability with respect to multicast route failure tolerance in K-connected MANET. We analyze the key issues namely connectivity, route failure tolerance and energy of the network. These issues can optimize the design of the routing protocol by means of cross layer interaction across the layers of the network. This paper analyzes the K-connected MANET for various parameters namely connectivity, no. of links, no. of spanning trees, no. of EDMSTs using graph theory metric, connectivity index.
\end{abstract}

\section{General Terms :}

Mobile ad hoc networks, Routing, Algorithms.

\section{Keywords:}

Edge disjoint minimum spanning trees, K-Connected MANETs, Connectivity Index and Route Failure Tolerance.

\section{INTRODUCTION}

A mobile ad hoc network (MANET) is an unusual type of wireless mobile network which forms a transitory network without the support of an established infrastructure or a centralized administration. MANETs are highly independent and self-organizing networks. Due to unpredictable and random mobility of the nodes, communication path tends to break in MANET. Therefore, the network survivability is a challenging issue especially in MANET. In this research work we address the survivability in terms of multicast route failure tolerance. The multicast route survivability or multicast route fault-tolerance of a network can be defined as an ability of the network to distribute multicast data packets correctly even though some multicast paths fail and is closely related to the connectivity of the network. For many network applications such as a sensor network/ ad hoc network for battlefield monitoring or wild fire tracking and etc., survivability is one of most important requirements, and therefore a number of related problems are proposed. It is significant to study connectivity, route failure tolerance and energy of the network to optimize the design of the multicast routing protocol by means of cross layer interaction across the layers of the network. This work analyzes these significant issues in $\mathrm{K}$ connected MANET using graph theory metric, connectivity index (CI).

\subsection{Related Work}

A lot of research has been done related to node density, critical transmission power and critical receiving power required for minimal connectivity of the MANETs [1] - [17]. The Number of neighbors needed for connectivity of wireless Networks is investigated by FENG XUE and P.R. KUMAR [18]. The analysis of a simple randomized scheme for constructing low-weight Kconnected spanning sub-graphs is given by [19].

There exist innovative routing MANETs protocols including optimizing the routing delays with minimum energy utilization, computing stable multicast paths, finding multiple multicast paths based on connectivity Index with bandwidth constraint, etc [20] [26]. However, the issue of rout failure tolerance, multipath multicasting in K-connected MANETs, which in turn affect the multicasting algorithms, needs to be studied.

This work focuses on study of connectivity, route failure tolerance and spanning tree analysis in K-connected MANET topologies. A $\mathrm{K}$-connected topology is one, in which, between any two nodes of the network, there are $\mathrm{K}$ independent routes, so that a very high rate of successful packet delivery can be made. The work has made significant observations and presented in the form of Lemmas which are novel to the best of our Knowledge.

The organization of this Paper is as follows: The Section 2 offers the network model and various definitions of interest. The Section 3 describes the study of K-connected networks using connectivity index. A novel EDMST algorithm to compute edge disjoint minimum spanning tree in K-connected networks is presented in the Section 4. The Section 5 brings the methodology adopted in the simulation and analysis of the result. The Section 6 concludes this paper.

\section{PRELIMINARIES}

\subsection{Network Model}

We study a network where nodes communicate with their neighbors using wireless links. The following assumptions are made: The neighborhood of a node is the set of nodes which can 
receive a packet transmitted by the nodes. Any packet transmitted by a node is received by all its neighbors. The source node of a multicasting is believed to know the entire information essential to construct the multicast tree. We use the term edge and link interchangeably. The cost of an edge from $u$ to $v$ is same as $v$ to $u$ where $(\mathrm{u}, \mathrm{v}) \in \mathrm{E}$.

\subsection{Definitions}

Some of the important definitions relevant to our discussion are given below.

- Complete graph(CG): A simple graph in which every pair of distinct vertices is connected by an edge. The complete graph on $n$ vertices and $n(n-1) / 2$ edges, and is denoted by $K_{n}$. It is a regular graph of degree $n-1$.

- Connected graph: A graph $G$ is said to be connected, if for every pair of vertices u, v belongs to $G$, there exist a path, otherwise $\mathrm{G}$ is disconnected.

- Tree: It is a graph without cycles.

- $\quad$ Spanning Tree of a graph (SPT): It is a spanning sub graph that is a tree.

- Minimum Spanning Tree (MST) of a Graph $G$ : Among all the spanning trees of a weighted graph $\mathrm{G}$, the spanning tree whose sum of edge weights is least is called MST of a graph G.

- $\quad$ Edge Disjoint Minimum Spanning trees (EDMSTs): Let E1 and E2 be the set of edges of minimum spanning tree-1(MST-1) and minimum spanning tree-2(MST-2) respectively, then MST-1 and MST-2 are said to be edge disjoint minimum spanning trees if $\mathrm{E} 1 \cap \mathrm{E} 2=$ Null Set [20] [24].

- K Node Multicast Minimum Spanning Trees (KMMST): A MST of graph G spanning K no. of multicast member nodes of the multicast group and forwarding nodes in $\mathrm{G}(\mathrm{FNG}), \mathrm{FNG} \subseteq V \mid-K$ [23].

- Energy of a Graph (GE): Let A be the adjacency matrix of a graph $G$. Let $\sigma \AA=\lambda_{1}, \lambda_{2}, \ldots \ldots . \lambda_{n}$ be the set of ordered eigen values of $A$. Then the energy $E_{\lambda}$ of the graph is defined as sum of absolute values of all the eigen values of $\mathrm{A}$, that is, $E_{\lambda}=\sum_{i=1}^{n}\left|\lambda_{i}\right|[\mathbf{2 6}]$.

- $\quad$ Network Energy (NE (t)): Let $P N_{i}(t)$ be the no. of packets transmitted by the node $i$ at time $t$ and TE is the transmission energy required to transmit one packet. Let $\mathrm{n}$ be the no. of nodes in the network at time t. The energy expenditure made by the node $i$ is the product of $P N_{i}(t)$ and TE. The energy of the network at any instant of time $t$ is the sum of energy expenditure made by each node of the network, that is, $\mathrm{NE}(\mathrm{t})=$ $\sum_{i=1}^{n} P N_{i}(t)(T E)[26]$.

- K-Connected Graph: A graph $G$ is said to be Kconnected, if on removal of any $\mathrm{K}$ nodes, the graph will become disconnected. The key property of interest relevant to routing in $\mathrm{K}$-connected graph is that there exist $\mathrm{K}$ disjoint paths between any two nodes of $\mathrm{G}$. A graph $\mathrm{G}$ is K-edge connected if it remains connected whenever fewer than $\mathrm{K}$-edges are removed. If a graph is K-edge connected then $\mathrm{K}<=\partial(G)$, where $\partial(G)$ is the minimum degree of any vertex $u \in V(G)$. We can write formally, a graph $\mathrm{G}$ with edge set $\mathrm{E}(\mathrm{G})$ is K-edge connected if $\mathrm{G} \backslash \mathrm{C}$ is connected for all $C \subseteq E(G)$ with cardinality of $\mathrm{C}<\mathrm{K}$.

- K-route failure tolerant (K-RFT): A route between any two nodes is said to have " $\mathrm{K}$ - route failure tolerant" if $\exists$ at least K-paths which are reliable without any failures.

- Graph Connectivity Index,$\Lambda(G)$ : Let $\mathrm{G}$ be such a graph. Denote by $(\mathrm{u}, \mathrm{v})$ the edge of $\mathrm{G}$, connecting the vertices $\mathrm{u}$ and $\mathrm{v}$. Then the connectivity index, also called Randi'c index or Randi'c weight or branching index [24] , of the graph G, proposed by Randic in 1975, we denote by $\Lambda(G)$ is defined as

$\Lambda(G)=\sum_{(u, v) \in E} \frac{1}{\sqrt{\partial(u) \partial(v)}}$

Vertices $(\mathrm{u}, \mathrm{v}) \in \mathrm{E}$, where $\partial(u)$ is degree of vertex $u \in V$.

\section{STUDY OF K-CONNECTED AD HOC NETWORKS USING CI}

The following Figures show examples of K-connected MANET for $\mathrm{K}=1,2$ and 3 . The analysis has lead to postulation of several Lemmas which are presented below. To the best of our knowledge Lemma-3 to Lemma-8 are novel.

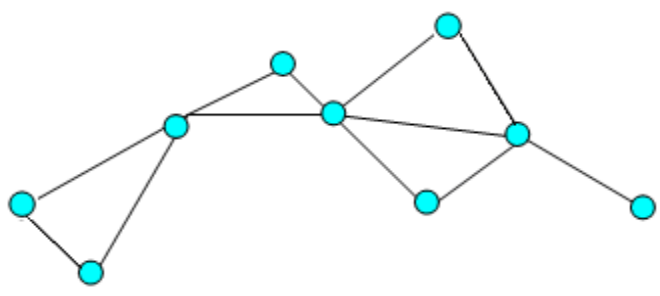

Figure 1 A One Connected MANET 


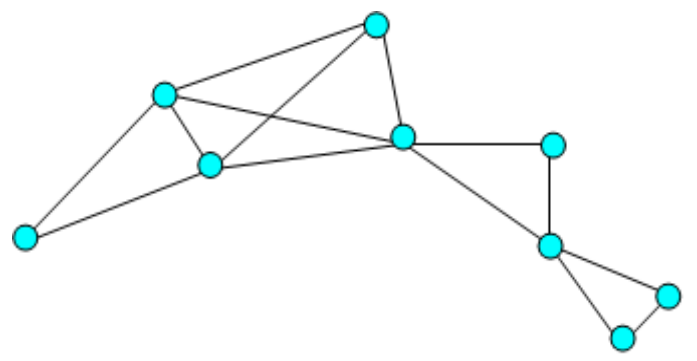

Figure 2 A Two Connected MANET

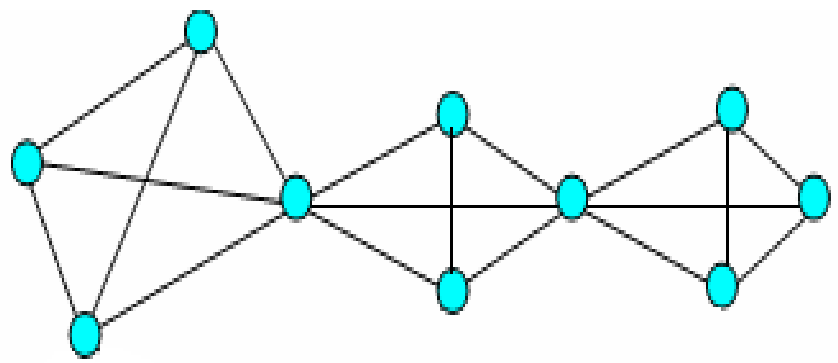

Figure 3 A Three Connected MANET

\section{Lemma-1 [26]}

For any positive integer $\mathrm{n}$, the minimum number of edges in a Kconnected graph is $\left\lfloor\frac{K(n)}{2}\right\rfloor$ for $\mathrm{K}>1$ and $(n-1)$ for $\mathrm{K}=1$.

\section{Lemma-2 (Bondy (1969)) [26]}

Let $\mathrm{G}$ be a graph with vertices $\mathrm{x} 1, \mathrm{x} 2, \ldots, \mathrm{xn}, \mathrm{d}(\mathrm{x} 1) \leq \mathrm{d}(\mathrm{x} 2) \leq \ldots$. $\leq \mathrm{d}(\mathrm{xn})$, where degree of the vertex denoted by $\mathrm{d}(\mathrm{x} 1)$. Suppose for some $K, 0 \leq K \leq n$, that $d(x j) \geq j+K-1$, for $j=1,2, \ldots$, $\mathrm{n}-1-\mathrm{d}(\mathrm{xn}-\mathrm{K}+1)$, then $\mathrm{G}$ is $\mathrm{K}-$ connected.

\section{Lemma-3}

A spanning tree with at least two vertices has at least two vertices of degree one and minimum connectivity index of the spanning tree is one.

Proof:

We know that a spanning tree is a connected network spanning all the nodes. Therefore, a spanning tree of two vertices should have at least one edge connecting them. That is they have minimum connectivity index,

$$
\Lambda(G)=\sum_{(u, v) \in E} \frac{1}{\sqrt{(1)(1)}}=1
$$

Hence the proof.

\section{Lemma-4}

A K-nodes multicast spanning tree is 1 - connected.

Proof:

We Know that a spanning tree of a connected graph $G$ with $n$ nodes can have (n-1) edges. Removal of any one node or any one edge in the spanning tree disconnects the graph $\mathrm{G}$ into sub graphs
G1 and G2. Therefore, according to the definition, a spanning tree is always 1-connected.

Further, a K node multicast spanning tree is also connected sub graph of the spanning tree. Therefore, from Lemma-4 it implies that a K-nodes spanning tree is also 1- connected.

\section{Lemma-5}

Every K-connected network is at most (K-1) route failure tolerant (RFT) where $r$ and $\mathrm{K}>1$.

Proof:

We Know that, if a graph is $\mathrm{K}$-connected, then there exist $\mathrm{K}$ disjoint paths between any two nodes (from a Corollary of Menger's Theorem). Here $K>1$, hence, the degree of every vertex in a connected network is always $>=\mathrm{K}$. We define that a route between any two nodes is said to be $\mathrm{m}$ - route failure tolerant if $\exists$ at least $\mathrm{m}$ reliable disjoint successful paths even if all the other $(\mathrm{K}-\mathrm{m})$ paths are failure, strictly $(\mathrm{m}<=\mathrm{K})$.

If all the paths are reliable we say that the K-connected graph is K-route failure tolerant. Assuming that at least one or more reliable paths exist, every $\mathrm{K}$-connected graph has at most (K-1) RFT network.

Let $(\mathrm{s}, \mathrm{d})$ be any two nodes in a K-connected network. Since G is $\mathrm{K}$-connected, $\exists \mathrm{K}$-disjoint paths between s and d. If all the (K-1) paths are failure, $\exists$ at least one path which is RFT. Hence G is RFT. If two disjoint paths are failure then (K-2) disjoint paths are available, hence $\mathrm{G}$ is (K-2) RFT.

Similarly, even if one such path is failure, we have (K-1) disjoint reliable path in that network. Hence G is (K-1) RFT at most.

In particular, if $\mathrm{G}$ happens to K-regular then the energy spent in transmitting packets between any two nodes can be optimized.

\section{Lemma-6}

The connectivity index (CI) of $\mathrm{K}$ regular and K-connected graph, $\mathrm{G}(\mathrm{V}, \mathrm{E})$ of $\mathrm{n}$ vertices is $=\frac{n}{2}$ for $\mathrm{K}>1$.

Proof:

From Lemma-1, the minimum number of edges required to construct K-connected graph $\mathrm{G}$ of n nodes is $\left\lfloor\frac{k(n)}{2}\right\rfloor$. Therefore, we can write, the CI of G, $\Lambda(G)=\sum_{(u, v) \in E}^{\left\lfloor\frac{k(n)}{2}\right\rfloor} \frac{1}{\sqrt{\partial(u) \partial(v)}}$.

Further, if $\mathrm{G}$ is $\mathrm{K}$-connected if and only if there are at least $\mathrm{K}$ edge disjoint paths between every pair of vertices. Therefore, every vertex is of minimum degree $\mathrm{K}$. Since $\mathrm{G}$ is $\mathrm{K}$-connected and $\mathrm{K}$ regular, degree of any node can be $\mathrm{K}$ only.

Therefore, $\Lambda(G)>=\frac{K(n)}{2} \frac{1}{\sqrt{\partial(K) \partial(K)}}$

$$
=\frac{K(n)}{2} \frac{1}{\sqrt{(K)(K)}}=\frac{n}{2} \text {. }
$$


Hence the proof.

\section{Lemma-7}

The CI of any two EDMSTs with (n-1) edges in a complete network of size $\mathrm{n}$ is same.

Proof:

The complete network graph (undirected) on $n$ vertices has $n$ ( $n$ 1)/2 edges, and is denoted by $K_{n}$. It is a regular network of degree $(n-1)$. Since degree of all nodes and number of edges (i.e., $n-1)$ of every spanning tree is same, connectivity index of every spanning tree has to be same. This is true even for edge disjoint spanning tree since the same vertex set (no. of vertices, no. of edges and their degrees are equal) is considered. Since number of vertices and their valence remains constant in every edge disjoint spanning tree, $\mathrm{CI}$ is also same and it is one [20].

\section{Lemma-8}

CI of any two EDMSTs in a K-regular K-connected network is same.

Proof:

We know that a $\mathrm{K}$-regular graph on $\mathrm{K}+1$ vertices has $\mathrm{K}(\mathrm{K}+1)) / 2$ edges, that is a complete graph. In a complete graph the connectivity index of any spanning tree is same and that is one. Since network is K-regular and K-connected, connectivity index of any edge is $\frac{1}{\sqrt{\partial(k) \partial(k)}}$. Therefore, we can write,

$$
\begin{gathered}
\Lambda(G)=\frac{k(k+1)}{2} \frac{1}{\sqrt{\partial(k) \partial(k)}} \\
=\frac{(k+1)}{2} .
\end{gathered}
$$

Hence the proof.

\section{ALGORITHMS}

This research work has used the algorithm given in section 4.1 based on Lemma-2 to find the connectivity of a K-connected MANET [26]. The Section 4.2 presents the novel algorithm devised and implemented as part of this research work to compute optimal edge disjoint minimum spanning trees.

\subsection{Algorithm to Compute K-Connectivity of MANETs}

Input:

Adjacency matrix of the given network. The $N \times N$ adjacency matrix $A=\left(a_{i, j}\right)_{N X N}$. of a given network is with $a_{i, j}=1$, whenever there exists a link between two nodes $i$ and $j$, otherwise 0 .

Out put:

Connectivity of the given network.

Steps: a) Compute the degree of the each node of the network by taking the row sums of $\mathrm{A}$. Row $R_{i}$ sum gives the degree of node i denoted by $d_{i}$.

b) Arrange the degrees of the node in ascending order.

c) Check for each node, whether $d\left(x_{j}\right) \geq j+K-1$, for $j=1 \ldots N-1-d\left(x_{N-K+1}\right)$.

The network is K-connected if all the nodes satisfy this condition.

\subsection{Algorithm to Compute EDMSTs Based on CI in K-Connected MANETs (KC-MANETs)}

In this Section, we present route failure tolerant quality of service multicast edge (link) disjoint minimum spanning trees protocol based on CI for KC-MANETs, called KC-EDMSTs algorithm. The protocol uses the following algorithm.

\subsubsection{Novel KC-EDMSTs Algorithm Based on CI for Multicasting}

Input: An undirected Graph, $\mathrm{G}=(\mathrm{V}, \mathrm{E})$ represented in terms of CI Adjacency (CIA) matrix of the given network. The $N \times N$ CI adjacency matrix, $A=\mathrm{a}(\mathrm{i}, \mathrm{j})$ of $N \times N$ of a given network is with $a(i, j)=$ connectivity index of vertices $(i, j)$, whenever two nodes $(\mathrm{i}, \mathrm{j})$ are connected by some edges and edge is associated with a connectivity index $>0$,otherwise zero.

Output: KC-EDMSTs table for K-connected MANET.

Steps:

a) Let Total-Edges be an integer variable initialized to zero, Total-Edges $=0$.

b) Let KC-EDMSTs be an empty table having no entries.

c) Using Prim's algorithm, compute the minimal spanning tree of the given network graph, $G$.

d) If minimal spanning tree does not exist, go to step $h$; else store the minimal spanning tree in the $K C$ EDMSTs table.

e) Compute Total-Edges $=$ Total-Edges $+(n-1)$.

f) If Total-Edges $>=|E|$ go to step $h$.

g) Compute $G=(V, E-\mathrm{e})$ where e is the edges of the minimal spanning tree, go to step $c$.

h) $S T O P$

\section{SIMULATION ANALYSIS AND RESULTS}

In order to study K-connected MANETs, we have simulated the relevant aspects of the network. In the simulation experiments algorithms devised are automated and significant results are noted. The simulation process starts with a minimal 1-connected network with $\mathrm{n}$ (which is fixed for each simulation) number of nodes and (n-1) edges. Then progressively, more links are added to built the K-connected MANET (maximum no. of links = n (n- 
1)/2 edges). The simulation process adopts the following methodology.

\subsection{Methodology}

The methodology has the following steps:

1. Choose two nodes randomly and add the links between them.

2. Calculate the CI of each edge and label the edges with their CI as weight.

3. Construct the network of size $n$.

4. Compute the $K$ connectivity of the network where $K=1$, $2,3, . .,(n-1)$ (using the Algorithm given in the Section 4.1).

5. Compute the CI of the entire network (sum of the connectivity index of all the edges in a network).

6. Compute the number of spanning trees in a KCMANETs using prim's algorithm.

7. Compute the KC-EDMSTs (using the Algorithm given in the Section 4.2)

8. Analyze the various parameters explicitly connectivity, no. of links, no. of spanning trees, no. of EDMSTs by plotting the graphs.

\subsection{Results}

The extensive numbers of simulations have been carried out to analyze number of issues including no. of links vs. connectivity, no. of spanning trees vs. connectivity and no. of EDMSTS vs. connectivity.

A MANET of 8 nodes is constructed and connectivity varied from 1 to 7 and corresponding no. of links in the network are noted. A graph drawn is shown in Figure 4 below, considering connectivity along $\mathrm{X}$-axis and number of links along Y-axis. It is observed that to construct the network of higher connectivity proportionate number of links is required. Figure 5 shows connectivity versus no. of spanning trees in a K-connected MANET. As connectivity of the network increases number of spanning tree increases.

Figure 6 and Figure 7 demonstrates that the effect of connectivity versus links, no. of EDMSTs in a KC-MANET and CI for a network size of 5 and 7 respectively. The simulation analysis has resulted in an important observation that as connectivity ( $\mathrm{K}$ value) increases, the number of links and connectivity index increases, and the no. of EDMSTs increases but not always. It is significant to recall that $\mathrm{K}$-connected network has $\mathrm{K}$ disjoint paths between any two nodes. If an edge $(\mathrm{u}, \mathrm{v})$ of an EDMST in KC-MANET fails, the network offers (K-1) alternate paths to reach from $\mathrm{u}$ to $\mathrm{v}$. Therefore, it is desirable to have higher $\mathrm{K}$ value network to offer high route failure tolerance.

A network of 5 nodes is constructed and its connectivity is varied from 1 to 4 . The no. of EDMSTs at different connectivity and their corresponding no. of links are noted and a corresponding graph is shown in the Figure 8. The simulation experiment is repeated by constructing a network of 7 nodes and result is shown in the Figure 9. From Figure 8 and 9, it can be noted that when a network of $\mathrm{n}$ nodes, $\mathrm{n}(\mathrm{n}-1) / 2$ edges, there exists maximum of (n-1) connectivity and no. of EDMSTs is equal to $n / 2$.
A random network of 50 nodes is built by varying no. of edges from 50, 100, 150.. 500. As no. of links varies graph energy varies which is shown in the Figure 10. A network of 50 nodes is constructed by selecting minimum no. of $\left\lfloor\frac{K(n)}{2}\right\rfloor$ edges with $\mathrm{K}$ value varied from 2 to 50 and its variation in graph energy is shown in the Figure 10. A spanning tree of the random network is computed when number of edges $=50,100,150 \ldots 500$. The variation in graph energy of the spanning tree is also shown in the Figure 10. It is observed that minimum graph energy of a $\mathrm{K}$ connected network is almost equals to graph energy of a spanning tree.

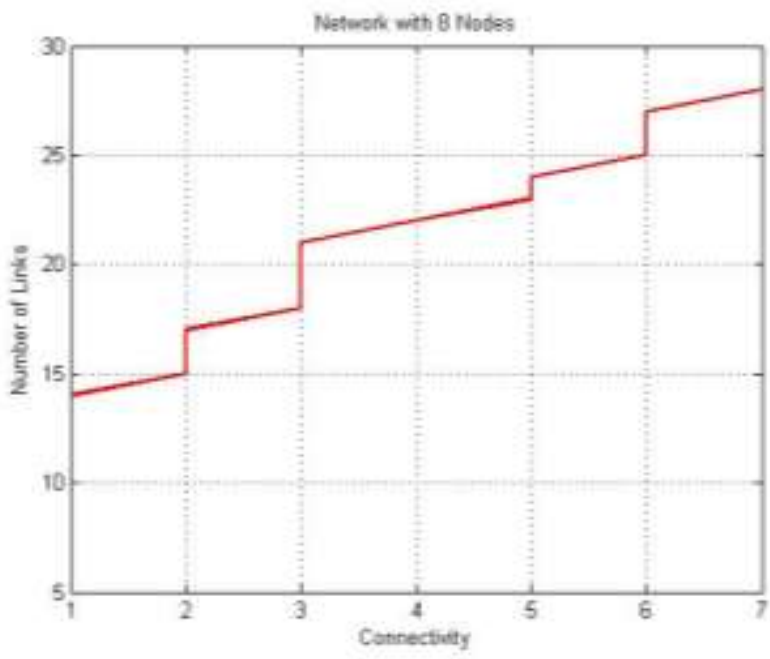

Figure 4 A Graph of Connectivity along X-axis and number of Links along $\mathrm{Y}$-axis

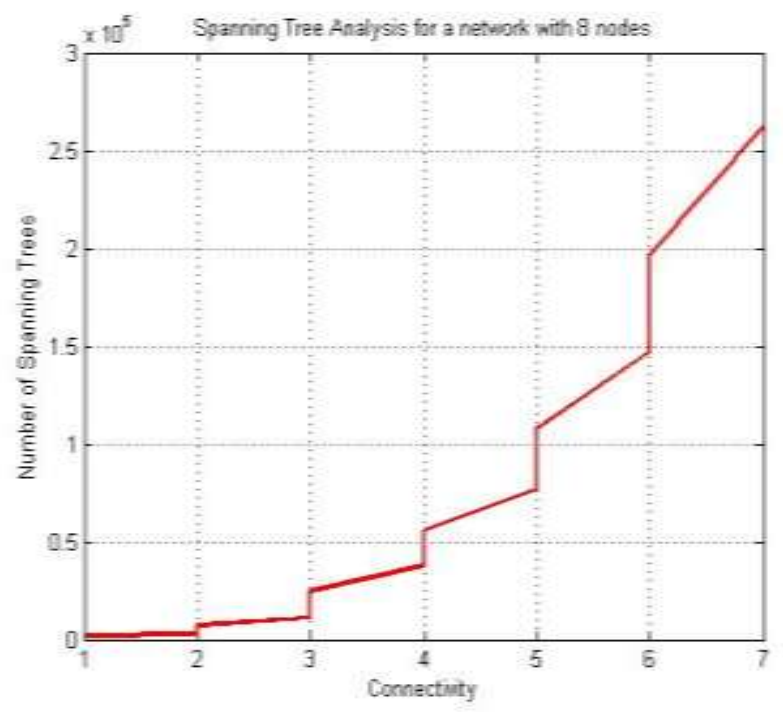

Figure 5 A Graph of Connectivity vs. no. of Spanning Trees in a K-connected MANET 



Figure 6 A Graph of Connectivity vs. no. of Links, no. of EDMSTs and CI.
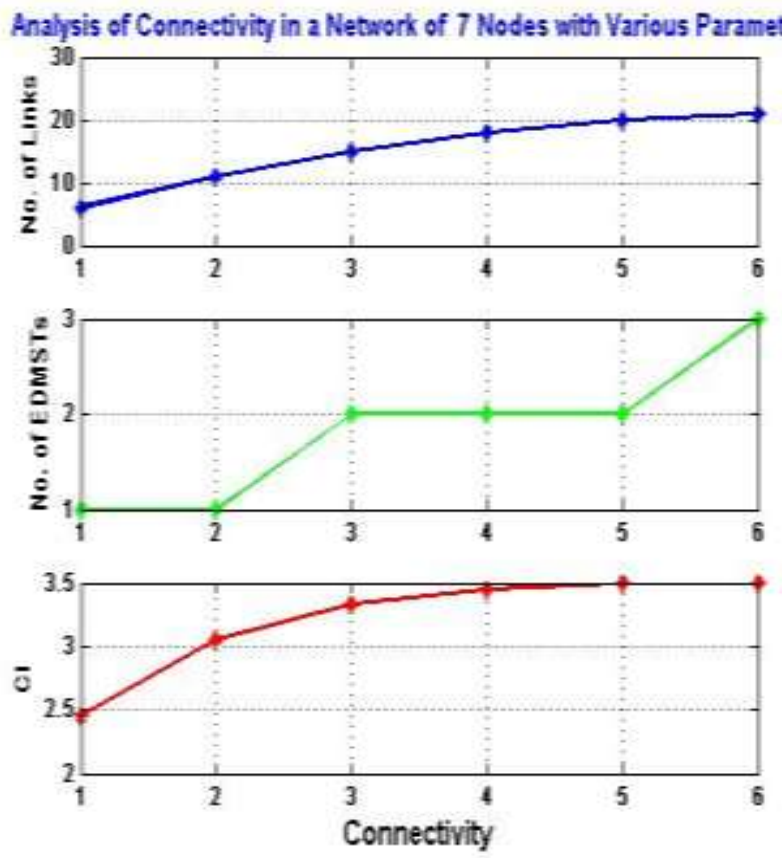

Figure 7 A Graph of Connectivity vs. no. of Links, no. of EDMSTs and CI

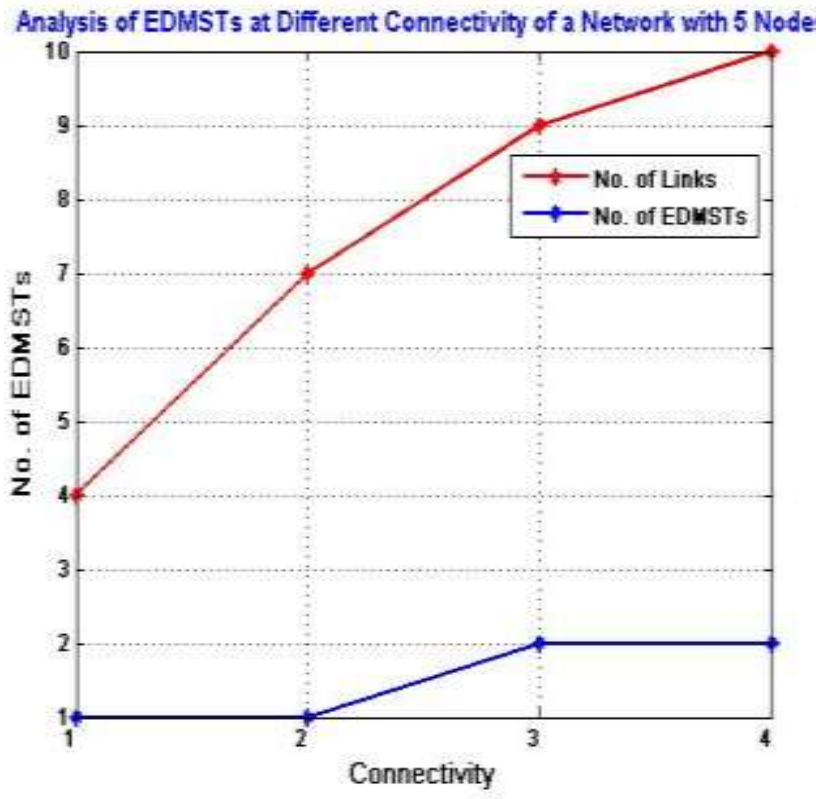

Figure 8 A Graph of Connectivity vs. no. of EDMSTs

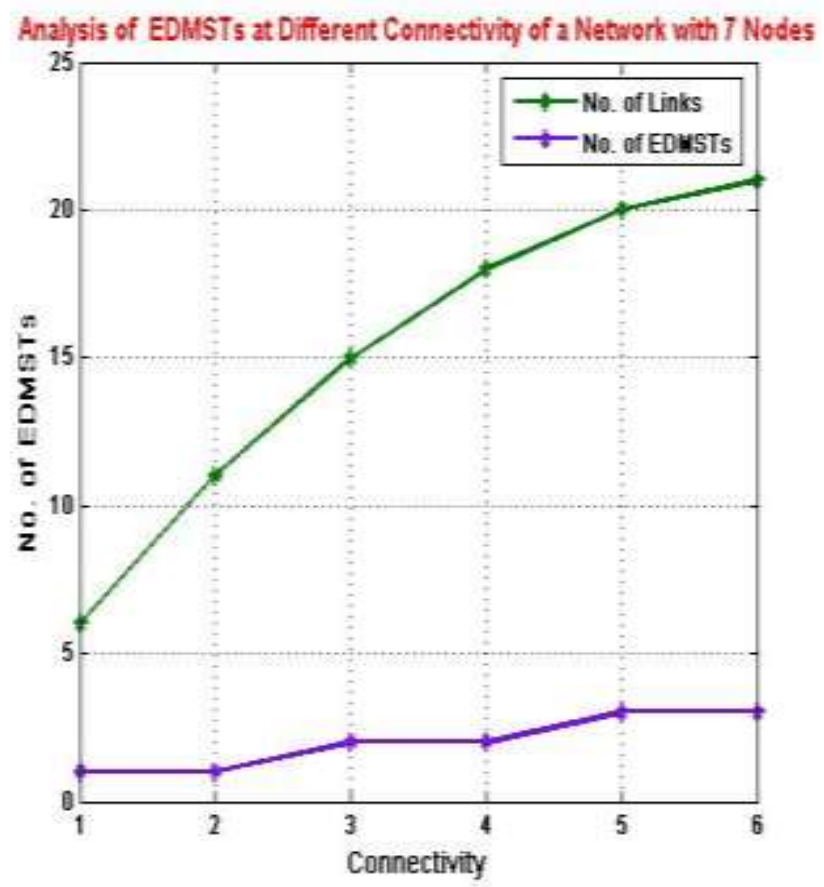

Figure 9 A Graph of Connectivity vs. no. of EDMSTs 


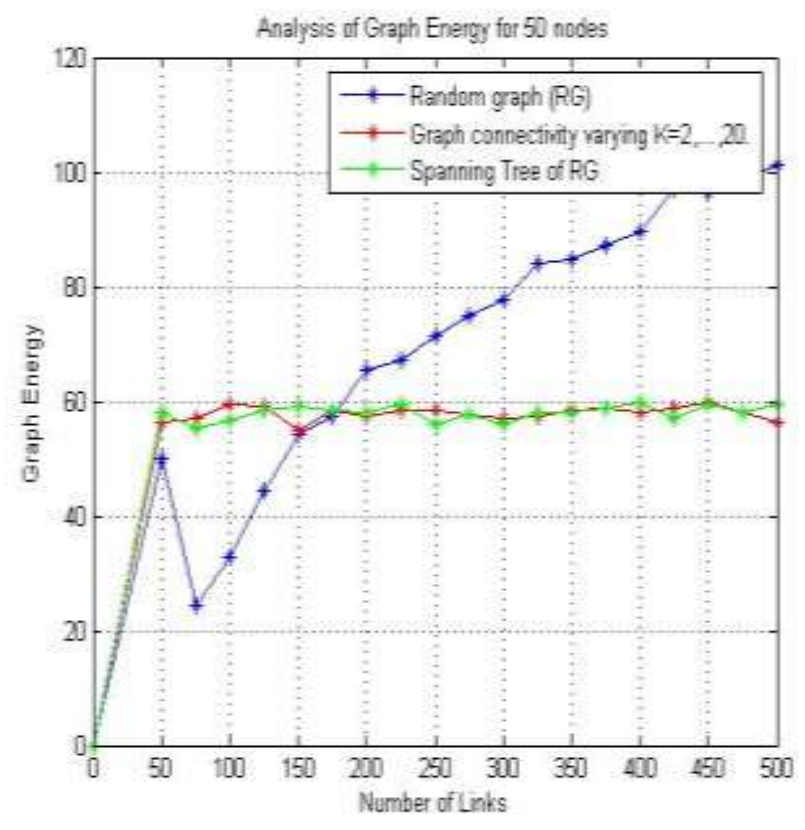

Figure 10 A Graph of no. of Links vs. Graph Energy

\section{CONCLUSION}

The study of K-connected network is motivated by the fact that when network is K-connected there is at least one route even if (K-1) routes fail. In multicasting applications if network is $\mathrm{K}$ connected, MANET offers robust, multipath multicasting with high packet delivery ratio. In this research work, the algorithms are devised and coded to compute number of spanning trees as well as EDMSTs in K-connected networks. We believe that there exists optimal number of multicast trees in K-connected network since any two nodes are connected by $\mathrm{K}$ disjoint paths. Further, if an edge between any two nodes fails, still multicasting can be continued as there are still (K-1) disjoint paths are reliable. In a $\mathrm{K}$ connected network, multipath multicasting could be achieved to yield all the advantages of multipath routing in MANET by adopting efficient load balancing policy. For example, an efficient load balancing policy diversifies the traffic among all possible multicast paths, even when primary path is available.

In this work we have investigated K-connected MANET using connectivity index as a metric to measure network connectivity. We have also analyzed spanning trees computation for different no. of nodes, no. of links and also varying connectivity. The investigation of various topological parameters, energy issues including graph energy and network energy are important inputs to the design of routing algorithms including multiple multicast paths, route failure tolerance and control of topology. The routing algorithms design shall have to note that increase in connectivity does not yield proportionate no. of disjoint multicast paths always (KC-EDMSTs).

\section{ACKNOWLEDGMENT}

All the authors express their gratitude to the Authorities of the Dravidian University, Kuppam, Andra Pradesh for the extensive support for the research activities in the Dept. of Computer
Science. Mr. Arun Kumar B.R further extends his special thanks to Sri Krishnadevaraya Educational Trust, Dr.Viresh K Basalalli, Dr.M.S. Indira, Prof. R.H.Nagabhusan and Dr.Rajeswari Sheshadri of Sir M Visvesvaraya Institute of Technology, Bangalore, Karnataka for their constant support and encouragement.. The lead author, Mr. Arun Kumar B.R extends his special thanks to Mr. Rajan, TCS, Bangalore.

\section{REFERENCES}

[1] R. Hekmat and P. Van Mieghem, "Study of Connectivity in Wireless Ad-hoc Networks with an Improved Radio Model", Delft University of Technology, Netherlands, http://www.wmc.ewi.tudelft.nl/ ramin/publications/connect. pdf.

[2] Pasi Lassila et al, "Connectivity Properties of Random Waypoint Mobility Model for Ad Hoc Networks", http://www.netlab.tkk.fi/tutkimus/ahras/publ/rwpconnmedhoc-crc.pdf.

[3] Christopher L. Barrett and D. Charles Engelhart, "Approximate Connectivity Graph Generation in Mobile Ad Hoc Radio Networks", http://csl.cse.psu.edu/publications/ ss03.pdf.

[4] Laura Marie Feeney, "Introduction to MANET Routing", Swedish Institute of Computer Science, http://www.nada. kth.se/kurser/kth/2D1490/05/lectures/feeney_mobile_adhoc _routing.pdf.

[5] Jon Kleinberg, "A First Random Graph Model", http://www.cs.cornell.edu/courses/ cs6850/2008fa/randomgraphs.pdf.

[6] Xiaowei Ying and Xintao Wu,"Graph Generation with Prescribed Feature Constraints", Univ. of North Carolina, http://www.siam.org/ proceedings/ datamining /2009/ dm09_088_yingx.pdf.

[7] Fan Chung and Linyuan Lu, "The Average Distance in a Random Graph with Given Expected Degrees", Internet Mathematics Vol. 1, No.1, pp. 91-114, http://www. internetmathematics.org/volumes/1/1/pp091_113.pdf.

[8] Brendan D. Mckay and Nicholas C. Wormald, Academy, Press, Inc 1990, http://cs.anu.edu.au/ bdm/papers /RandRegGen.pdf.

[9] K.V.R.H. Bharadwaj and V. Sunitha, "Application of Random Graph Theory and Percolation Theory in MANETs", $\quad$ http://magnet.daiict.ac.in/ magnet_members/Btech/2004/ KVRHBharadwaj/report.pdf.

[10] Clement Fredembach and Graham D. Finlayson, "Hamiltionian path based shadow removal", School of Computing Sciences, University of East Anglia, Norwich.

[11] M. Didi Biha and A. R. Mahjoub, "The k-edge connected subgraph problem I: Polytopes and critical extreme points", Linear Algebra and its Applications, Vol. 381, PP. 117-139, April 2004, pp. 117-139.

[12] Angela Mestre and Centro de Fisica Teorica, "Generating connected and 2-edge connected graphs", Journal of Graph Algorithms and Applications,http://jgaa.info/ Vol. 13, No. 2, PP. 251-281, 2009.

[13] Harold N. Gabow, "Approximating the Smallest k -Edge Connected Spanning Subgraph by LP-Rounding”, Published online in Wiley InterScience (www.interscience.wiley.com), Wiley Periodicals, Inc. 
[14] Jorgen Bang Jensen and Stephan Thomasse, "Highly connected hypergraphs containing no two edge-disjoint spanning connected subhypergraphs", Department of Mathematics and Computer Science, University of Southern Denmark, Denmark.

[15] Andras Frank and Zoltan Kiraly,"Parity Constrained k-EdgeConnected Orientations", Springer, Vol. 1610/1999.

[16] Bill Jackson and Kiyoshi Yoshimoto,"Spanning Even Subgraphs of 3-edge-connected Graphs", University of London, March 2006.

[17] Maleq Khan, Gopal Pandurangan and V.S. Anil Kumar, "A Simple Randomized Scheme for Constructing Low-Weight k-Connected Spanning Subgraphs with Applications to Distributed Algorithms".

[18] FENG XUE and P.R. KUMAR, "The Number of neighbors needed for connectivity of wireless Networks", Wireless Networks, 169-181, 2004, Kluwer Academic Publishers.

[19] Maleq Khan, Gopal Pandurangan and V.S. Anil Kumar, "A Simple Randomized Scheme for Constructing Low-Weight k-Connected Spanning Subgraphs with Applications to Distributed Algorithms".

[20] Arun Kumar B. R, Lokanatha C. Reddy, Rajan and Prakash S. Hiremath, " Route Failure Tolerant Multicast In Mobile Ad Hoc Networks Using Disjoint Minimum Spanning Trees", the second IEEE ICCSIT 2009, Aug. 8 - 11, 2009, Beijing, China.
[21] F. Harary, Graph Theory, Narosa Publishing House.

[22] Patil et al, "Resource Allocation Using Multiple EdgeSharing Multicast Trees", Vehicular Technology, IEEE Transactions on Sept. 2008 Vol. 57, PP. 3178 - 3186.

[23] Arun Kumar B. R, Lokanatha C. Reddy, Prakash S. Hiremath and Rajeswari Sheshadri, "K-Nodes Multicasting Minimum Cost Spanning Trees In Wireless Mobile Ad Hoc Network (MANET)", International Journal of Computer Applications [IJCA],www.ijcaonline.org/archives/number4/110-225.

[24] Arun Kumar B. R, Lokanatha C. Reddy, and Prakash S. Hiremath, "Route Failure Tolerant Multicast in Mobile Ad Hoc Networks Using Disjoint Minimum Spanning Trees Based on Connectivity Index with Bandwidth constraint", IEEE 2nd International Conference on Advance Computing Conference, IACC 2010, Tharpur University, Patiala147004, Punjab.

[25] Ken-ichi Kawarabayashi and Yusuke Kobayashi, "The edge disjoint paths problem in Eulerian graphs and 4-edgeconnected graphs", http://www.siam.org/proceedings/soda /2010/SODA10_029_kawarabayashik.pdf.

[26] M.A.Rajan, M.Girish Chandra, Lokanatha C. Reddy and Prakash S. Hiremath, "Topological and Energy Analysis of K- Connected MANETs: A Semi-Analytical Approach", IJCSNS International Journal of Computer Science and Network Security, Vol.8, No.2, PP.199-207, Feb. 2008. 